\title{
Intravenous Thrombolysis in a Rare Case of Bilateral Medial Medullary Infarction
}

\author{
Julie Anne V. Gamboa, MD ${ }^{\mathrm{a}}$, Grace Kathleen T. Serrano, MD ${ }^{\mathrm{b} *}$ \\ a jasvgamboa@gmail.com \\ ${ }^{\mathrm{a}, \mathrm{b}}$ Makati Medical Center, No. 2 Amorsolo Street, Legaspi Village, Makati City, Philippines 1229
}

\section{Abstract}

Based on our PuBMed and Cochrane search, this is the first case report that describes the clinical outcome of thrombolysis in a rare case of bilateral medial medullary infarction.

The objective of this paper is to describe the outcome of thrombolysis in a frequently devastating bilateral medial medullary infarction.

A 37-year-old Chinese male was seen at the emergency department four hours from symptom onset of sudden and rapidly progressing bilateral motor weakness with associated dizziness. The patient was seen awake, not in cardiorespiratory distress. Pupils were 3 millimeters isocoric and briskly reactive to light. Extraocular muscle movements were full and equal and there was torsional nystagmus on all gazes. There was right central facial palsy and weak gag reflex bilaterally. Tongue was midline on protrusion. Motor strength test revealed right sided hemiplegia and left sided hemiparesis (2-/5 on MMT). Bilateral Babinski sign was elicited. There was no sensory deficit to light touch. Magnetic resonance imaging of the brain showed acute infarction of the bilateral anteromedial medulla.

Alteplase was given at a dose of $0.7 \mathrm{mg} / \mathrm{kg}$ was given at exactly 4.5 hours post-ictus. There was no complication post-rtPA. He was discharged on the 12th day post-ictus, still with dizziness but with improved muscle strength on all extremities to 3/5 MMT except for the right arm which remained paralyzed. He was able to tolerate oral feeding and had a Modified Rankin Scale score of 4.

Keywords: thrombolysis; rtPA; clinical outcome; posterior circulation; bilateral medial medullary infarction

\section{Introduction}

The triad of medial medullary syndrome consists of hypoglossal paralysis, contralateral hemiparesis sparing the face, and contralateral loss of deep sensation. Medial Medullary Infarction (MMI) accounts for only $0.5 \%$ - 
$1.5 \%$ of all strokes while bilateral MMI is even rarer at less than $1 \%$. Bilateral MMI are usually diagnosed at autopsy and the rate of diagnosis by neuroimaging is still low. ${ }^{1}$ Thirty percent of patients do not have a lesion in initial DWI-MRI and diagnosis is usually made from history and clinical examination. ${ }^{2}$ Intravenous thrombolysis is a standard treatment for both anterior and posterior circulation ischemic stroke. However, posterior circulation ischemic strokes are under-represented among randomized control trials on intravenous thrombolysis. ${ }^{3}$ This study discusses a case of a patient with Bilateral MMI who underwent thrombolysis.

\section{Case Presentation}

A 37-year-old Chinese male presented with sudden rapidly progressing right sided weakness followed by left sided weakness with associated dizziness and vomiting four hours prior to arrival. The patient was a smoker and had no known co-morbidities. Initial blood pressure was 120/70. Heart rate was regular. He was afebrile and was not in cardiorespiratory distress. Oxygen saturation at room air was $97 \%$. The patient was awake and verbally responsive. Neurological examination was limited due to a language barrier. Pupils were 3 millimeters isocoric and briskly reactive to light. Extraoccular muscle movements were full and equal and there was torsional nystagmus on all gazes. There was right central facial palsy and weak gag reflex bilaterally. Tongue was midline on protrusion. Manual motor testing revealed 0/5 muscle strength on right sided extremities and 2-/5 muscle strength on left sided extremities. Bilateral Babinski sign was elicited. There was no sensory deficit to light touch. Proprioception, vibration, pain and temperature senses were not tested. A stat MRI of the brain revealed "heart-shaped" restricted diffusion signals at bilateral anteromedial medulla with corresponding signal drop in apparent diffusion coefficient sequence (as shown in Figure 1). MRA of the intracerebral and neck vessels were unremarkable. Other investigation findings revealed normal lipid profile, normal ESR at $3 \mathrm{~mm} / \mathrm{hr}$. High sensitivity CRP was elevated at $1.43 \mathrm{mg} / \mathrm{dl}$ and antinuclear antibody (ANA) was positive at 1: 640 serum dilution. Further rheumatologic work-up was deferred due to financial constraints.

A total of $50 \mathrm{mg}$ or $0.7 \mathrm{mg} / \mathrm{kg}$ of intravenous recombinant tissue plasminogen activator (rTPA), Alteplase, was given at exactly 4.5 hours post-ictus.

Twelve hours after administration of thrombolysis, motor strength improved to $1 / 5$ on the right upper extremity but remained $0 / 5$ on right lower extremity. The left sided extremities remained $2 / 5$. There was persistence of nystagmus, but it became less pronounced. Aspirin $80 \mathrm{mg}$ daily was started after a repeat cranial CT scan 24 hours post-thrombolysis showed absence of hemorrhage. The patient had mild oxygen 
desaturations to $90-92 \%$ initially requiring $\mathrm{O} 2$ support with nasal cannula, but this spontaneously resolved. The patient did not require NGT insertion and was able to swallow without aspiration. He was discharged after 12 days with improved muscle strength, $3 / 5$ on both lower extremities and distal left upper extremity. However, the right upper extremity strength remained 0/5. Discharge mRs score was 4.

\section{Discussion}

Our patient presented with bilateral weakness, nystagmus, and facial palsy. According to a systematic review, the most common clinical presentation for MRI-proven bilateral MMI is bilateral motor weakness (64.9\%), followed by dysarthria (48.6\%) and nystagmus (48.6\%), hypoglossal palsy (40.5\%), respiratory failure $(24.3 \%)$, dysphagia $(16.2 \%)$ and facial palsy $(16.2 \%){ }^{1}$

The "heart appearance" sign - termed so because of its shape which is the characteric MRI finding in bilateral MMI - was also apparent in the patient's DWI and ADC MRI sequences done four hours from symptom onset. ${ }^{4,5,6,7}$ Repeat neuroimaging is usually necessary to document bilateral MMI. In a clinical review, 3 out of 8 patients (37.5\%) with MMI did not show MRI lesion on the day of onset of symptoms. Only the second set of DWI images confirmed the lesions. ${ }^{4}$

The proposed explanation for the occurrence of simultaneous bilateral MMI is related either to extended thrombosis in the vertebrobasilar junction, anatomic variability of perforator branches that supply the medulla, or variation of ASA originating from a vertebral artery. ${ }^{1}$ When comparing MMI and lateral medullary infarction (LMI), atherosclerosis was found to be more common in MMI (87.5\%) than in LMI (58\%). Our patient had normal intracerebral and neck vessels by MR angiography.

The present case illustrates a good outcome following thrombolysis in a patient with Bilateral MMI. Our patient showed recovery over the course of his 12-day hospital admission. Research points to the importance of early intervention in optimizing patient outcomes. In three recent case reports, patients with heart-shaped medullary stroke who did not receive thrombolysis due to late consult ( 1 to 4 days from onset) exhibited poor outcomes. These included dysarthria, quadriplegia and deterioration of motor function, bilateral facial weakness, and a need for nasogastric feeding, which persisted upon follow-up for two patients. ${ }^{5,6,7}$

There is evidence of better clinical outcomes for thrombolysis after 3 months as well as lower NIHSS scores at 2-hours and 24-hours post-thrombolysis for patients with posterior circulation ischemic stroke compared to 
those with anterior circulation ischemic stroke. ${ }^{3}$ One case report describes the effectiveness of thrombolysis in treating MRI-negative anterior spinal artery ischemic stroke. The patient was given a dose of Alteplase $(0.9$ $\mathrm{mg} / \mathrm{kg}$ ) 4.5 hours after the symptom onset. Although he exhibited initial paralysis, he was able to regain some function in his legs and arms after four months, rendering him able to walk with a stick. ${ }^{8}$ This suggests that thrombolysis may have long-term clinical benefit on functional recovery even in MRI-negative posterior circulation stroke.

\section{Conclusion}

Thrombolysis was a safe treatment modality for our patient. Minimal improvement of motor weakness and nystagmus was seen 12 hours post-thrombolysis. Further motor improvement was seen within 12 days postictus. There was no deterioration to respiratory failure and the patient was able to tolerate oral feeding. A larger study on Bilateral MMI with longer follow-up period is recommended to further elucidate the benefit of thrombolysis in this type of stroke.

\section{Author Artwork}
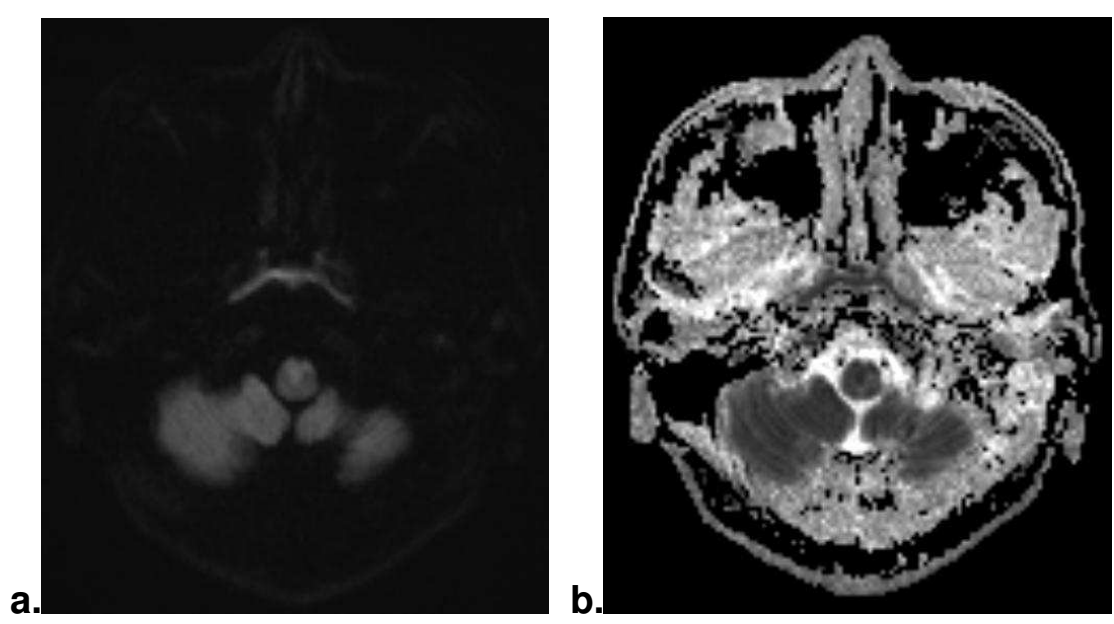
C.
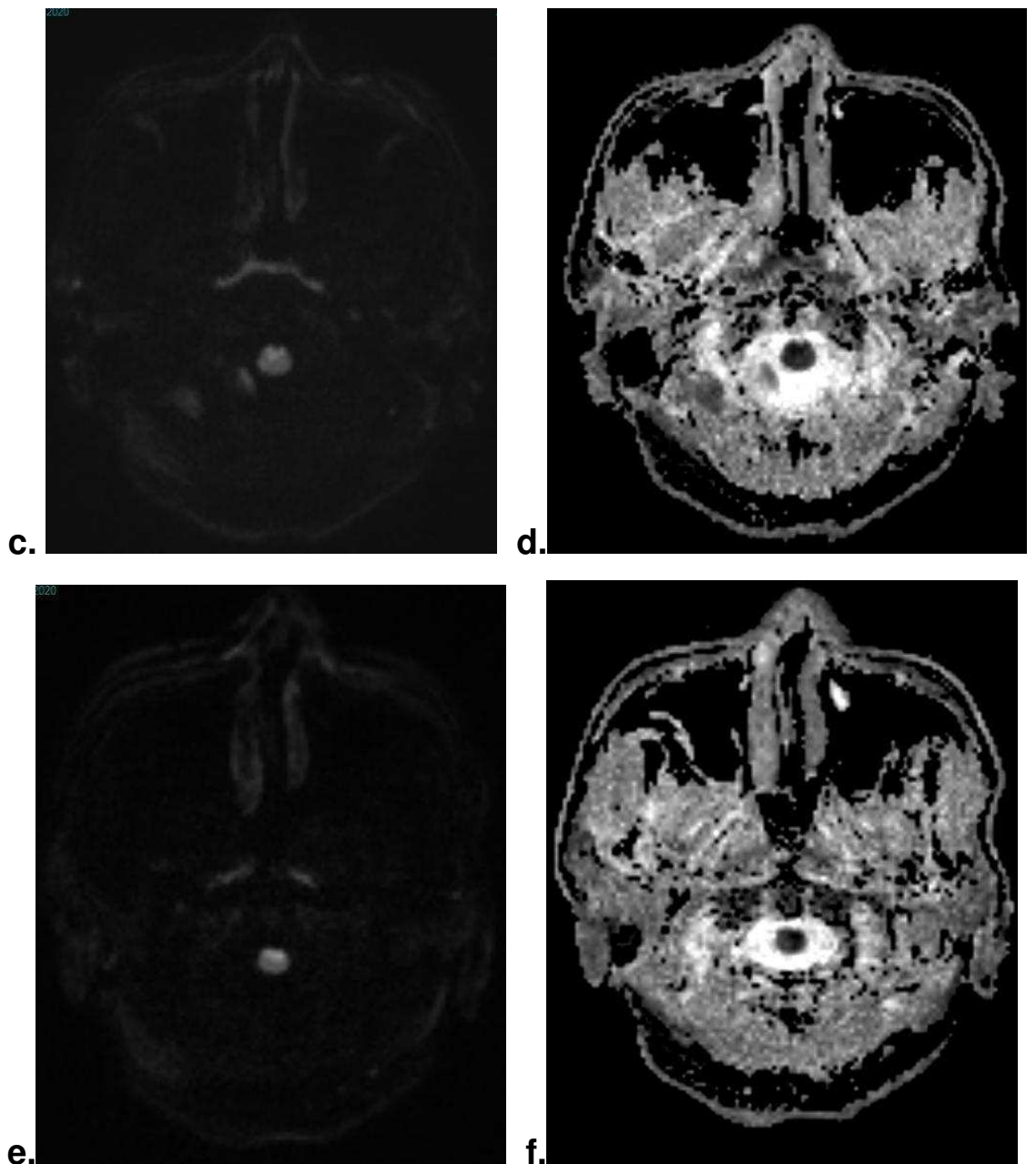

Figure 1. (a, c, e) Plain Brain MRI: diffusion weighted images; (b, d, f); Plain Brain MRI: apparent diffusion coefficient images

\section{References}

Pongmoragot, J., Parthasarathy, S., Selchen, D., \& Saposnik, G. (2013). Bilateral Medial Medullary Infarction: A Systematic Review. Journal of Stroke and Cerebrovascular Diseases, 22(6), 775-780. doi:10.1016/j.jstrokecerebrovasdis. 2012.03.010

Forshing Lui; Prasanna Tadi; Arayamparambil C. Anilkumar. (2020) Wallenberg Syndrome. Stat Pearls. https://www.ncbi.nlm.nih.gov/books/NBK470174/

Dorňák, T., Král, M., Šaňák, D., \& Kaňovský, P. (2019). Intravenous Thrombolysis in Posterior Circulation Stroke. Frontiers in Neurology, 10. doi:10.3389/fneur.2019.00417

Fukuoka, T., Takeda, H., Dembo, T., Nagoya, H., Kato, Y., Deguchi, I., Tanahashi, N. (2012). Clinical Review of 37 Patients with Medullary Infarction. Journal of Stroke and Cerebrovascular Diseases, 21(7), 594-599. doi:10.1016/j.jstrokecerebrovasdis.2011.01.008

Krishnan, P. (2020). Heart in the brain: classical imaging sign in bilateral medial medullary infarction. British Journal of Neurosurgery, 1-2. doi:10.1080/02688697. 2020.1765978 
Zhi-Hua Zhou, Yun-Fan Wu, Wei-Feng Wu, Ai-Qun Liu, Qing-Yun Yu, Zhong-Xing Peng, Ming-Fan Hong (2020). Giant "heart appearance-like sign" on MRI in bilateral ponto-medullary junction infraction: case report. BMC Neurol. 2020 Mar 23;20(1):107. doi: 10.1186/s12883-020-01683-7

Jiang, S., Wang, L., Yan, Y., Zhu, Q., Wan, J., Sun, J., \& Wu, B. (2020). Fabry disease presenting as bilateral medial medullary infarction with a "heart appearance" sign: a case report. BMC Neurology, 20(1). doi:10.1186/s12883-020-01766-5

Muller, K. I., Steffensen, L. H., \& Johnsen, S. H. (2012). Thrombolysis in anterior spinal artery syndrome. Case Reports, 2012(sep05 2), bcr2012006862-bcr2012006862. doi:10.1136/bcr-2012-006862

Kim, J. S., \& Han, Y. S. (2009). Medial Medullary Infarction: Clinical, Imaging, and Outcome Study in 86 Consecutive Patients. Stroke, 40(10), 3221-3225. doi:10.1161/strokeaha.109.559864 